\title{
Structure and Science
}

\author{
Marvin L. Birnbaum, MD, $\mathrm{PhD}$
}

Should we force science down the throats of those who have no taste for it? It is our duty to drag them kicking and screaming into the twenty-first century. I am afraid that it is.

Sir George Porter, Speech 1986

Discovery consists of seeing what everybody has seen and thinking what nobody has thought.

Albert von Szent-Gyorgyi,

In Good: The Scientist Speculates, 1962

In a summary of the WHO-sponsored meeting in Phuket regarding the Earthquake and Tsunami that devastated parts of nine countries in southeast Asia (December 2004), Dr. David Nabarro asked: "Why have we not learned from what we have learned?". This message continues to be relevant and the reasons that we continue not to learn are becoming increasingly clear. We continue to rely on unstructured reports where critical information is lost in the absence of structure. Without a structure, it is difficult to integrate what has been observed into plans and the interventions that result from such plans.

Although many "good" studies have been published relevant to disaster and emergency health, without structure, this information is difficult and often impossible to access. It remains difficult, at best, to integrate the findings from multiple studies. Unlike the data and information obtained in experimental or longitudinal, controlled studies that utilize quantitative data collection methods and the mandatory requirements for reporting the results, much of the information collected in disaster and emergency health preparedness and response is qualitative. Hence, it is difficult to classify the information, and without categorization, the information remains difficult to access. Without a standardized structure, it is not possible to validate the findings through comparisons with other similar studies.

A structure has three main components: (1) a foundation that supports the superstructure; (2) a framework that holds the structure together and provides the environment for the processes that make up the functions of the structure; and (3) components that convert the framework into a functional entity-the physical components that are essential in order for the production processes that operate within the structure.

The foundation of all sciences is the language used in support of the science. Without universally accepted terminology, no science can be built. Therefore, the foundation of disaster and emergency health must be the terminology upon which it is constructed. Unfortunately, amidst all of the work done so far, there is no common, comprehensive terminology upon which to base the science of disaster and emergency health. There are a host of glossaries of terms used today - each used by different organizations, and there are many terms for which the definitions are diverse. Each organization proposes that its definitions are the correct ones. Without uniform definitions, communication does not occur. When the terms used in reporting the results from research are not understood, confusion reigns and important findings are lost, preventing the use of these terms in the interpretation of future events. We have been able to pick and choose those definitions that we believe best meet our needs. This weakness in the foundation renders even the most carefully constructed studies difficult to understand and threatens our science. The ability to support the findings that relate to the science and integrate them into standards and best practices is difficult. Without a glossary of terms agreed on by all of the stakeholders, there cannot be a solid science. In order to build our science on a firm foundation, a consensus conference on disaster health terminology must be convened without delay and the resulting glossary must be accepted by each of the stakeholders.

The framework that holds the structure together includes the parts of the structure that make the functioning elements of the structure possible. For a hospital, the framework consists of the framing of the building and the rooms within it, including the steel girders, reinforced concrete, and elements that stabilize it during events for which it is at risk. Functions depend upon the appropriateness of the framework in which they are to be conducted. In terms of disaster and emergency health, and especially for framing the results of evaluations and research that comprise the science, specific frameworks are required to support the functions. All of the science must be set into a framework that allows the comparison of findings to validate and collate the findings within the structure.

Currently, there is not a universally accepted framework into which to force our research. This impairs our ability to weld the results of the research into concepts upon which best practices are based. Our practices generate theories that lead to future research questions. The absence of a universal framework for disaster research results in unstructured reporting of important research and difficulty in validating the findings and building a science for disaster health.

The third part of the structure consists of all of the accoutrements (furniture, equipment, and supplies) required for the production functions that are carried out by those that utilize the space provided by the framework. In order for a hospital to continue to function, it is essential 
that this part of the structure remains productive. For example, although the laboratory framework has sustained the forces released by an event, if the equipment within it is rendered non-functional, the laboratory cannot continue to function. Thus, it is not just the foundation and framework that must absorb the energy, but the equipment and supplies, essential for continued function, must be operational.

In terms of the ability to design, conduct, and report research, certain accoutrements also are essential. For example, using appropriate indicators of function. Appropriate indicators of function are critical elements for structuring research in a way that permits integration into the concepts that drive best practices. When different indicators are employed to express the same phenomenon, it is not possible to compare the results obtained with other similar stud- ies. Therefore, the results may be lost in the deep hole that has consumed the results from unstructured reports of important research efforts.

We cannot continue to conduct and report important research in an unstructured way. We must examine the structures that are used and agree on a basic structure for designing and reporting our labors. We must agree on the language that we use, the framework used to design and report our work, and on the indicators that best reflect our ability to cultivate our science.

A theory can be proved by experiment, but no path leads from experiment to the birth of a theory.

Albert Einstein, in Mackey, The Harvest of a Quiet Eye, 1977

Science may be described as the art of oversimplification.

Sir Karl Popper,

The Observer, 1982

\section{References}

1. Nabarro D: Putting it together:Stronger public health capacity within disaster management systems. Prehosp Disast Med 2005;20(6):483-485. 